\title{
PROFILING COMPUTER ENERGY CONSUMPTION ON ORGANIZATIONS
}

\author{
Rui Pedro Lopes, Luis Pires, Tiago Pedrosa, Vasile Marian \\ Politechnic Institute of Braganca, Braganca, Portugal \\ rlopes@ipb.pt,luica@ipb.pt,pedrosa@ipb.pt,a17591@alunos.ipb.pt
}

Keywords: Energy saving, power management, policy-based consumption optimization

\begin{abstract}
Modern organizations depend on computers to work. Text processing, CAD, CAM, simulation, statistical analysis and so on are fundamental for maintaining high degree of productivity and competitiveness. Computers in an organization, consume a considerable percentage of the overall energy and, although a typical computer provides power saving technologies, such as suspending or hibernating components, this feature can be disabled. Moreover, the user can opt for never turning off the workstation. Well defined power saving policies, with appropriate automatic mechanisms to apply them, can provide significant power savings with consequent reduction of the power expense.

With several computers in an organization, it is necessary to build the profile of the energy consumption. We propose installing a software probe in each computer to instrument the power comsumption, either diretly, by using a power meter, or indirectly, by measuring the processor performance counters. This distributed architecture, with software probes in every computer and a centralized server for persistence and decision making tries to save energy, by defining and applying organization level power saving policies.
\end{abstract}

\section{INTRODUCTION}

Many organizations depend heavily on computers and other information technology equipment to work. These resources consume a significant amount of energy, contributing to the overall power expense.

Typically, computer equipment act in two roles: as a server, providing remote access to network applications, such as network file systems, web servers, e-mail and other, or as a desktop workstation, where the user runs graphical applications and tools. The energy consumption is different in both situations, and depend on the energy necessary to power the device, and the use pattern.

The first is conditioned by the technology used in the computer components, such as the CPU, network interface card, hard disk as well as the monitor. Moreover, Power Management techniques, such as ACPI (Hewlett-Packard et al., 2006), allow a considerable reduction of power consumption. The use pattern is related to how the user interacts with the computer, it's operating system and applications.
There is a high degree of uncertainty in predicting the energy consumption for both approaches. Power Management is not always used, either because the equipment does not support it or because it is disabled in the operating system, the user behaviour in relation to turn-off rates is erratic, and the type and quantity of equipment in use at the office varies randomly.

Because of this uncertainty, the optimization of computer energy consumption has to act on two axis: a) enforcing power saving policies and b) appealing to user behaviour.

\section{COMPUTER ENERGY CONSUMPTION}

The energy used by a computer depends on two different aspects: the energy required to run the device (power draw) and how the computer is used (use pattern) (Webber et al., 2005). 


\subsection{Power Draw}

The power used by a computer is in direct proportion to the energy required by it's components. The memory system, composed of the main memory and system caches consumes a significant percentage of energy, tending to increase with the cache size and main memory. Several authors address this issue by, for example, optimizing the address translation and compiler techniques for energy saving (Ashok et al., 2004). Other proposals seek to optimize the power state of memory as a function of the memory load (Diniz et al., 2007) or by transiting devices to low power after unused for some time (Li et al., 2004).

Similar to the previous case, hard disk drives can also be forced into a low power state if unused for some time (Li et al., 2004). Some approaches adapt the compiler to extend, as much as possible, the time between disk accesses to allow switching the disk into a hibernate state, thus saving energy (Hom and Kremer, 2005). The insight into how disk drives use the energy can also provide valuable information related to power consumption (Zedlewski et al., 2003).

There is a large variety of computer processors on the market nowadays and the fact is that every series that is being produced has a specific hardware design and implementation that affects the energy consumption. Designing processors that can perform tasks efficiently without overheating is a major design factor for many CPU manufacturers. In a desktop computer, the CPU is one of the prominent energy consumer component and successfully reducing it can represent a considerable slice of the overall machine consumption.

The measurement of the consumed energy can be performed in real-time, either directly (by using a power meter) or indirectly, by using the CPU's performance counters (Isci and Martonosi, 2003). With proper clock speed control associated to an adequate scheduling of processes, it is possible to reduce the CPU power consumption (Weiser et al., 1996). Reducing the clock speed allows lowering the supply voltage, which can significantly reduce power dissipation (Hsu and Kremer, 2003).

\subsection{Use Pattern}

The randomness of the user behaviour in relation to turn-off rates leads us to consider an automatic procedure to turn off the computer based on specific policies. (Webber et al., 2005) shows that users turn-off rates range from $0 \%$ to $91 \%$, for example. On a different study, using stickers to remind the user to turn off the computer when not in use had some effect.
However, after some weeks, the percentage diminished (Newsham and Tiller, 1994). Only automatic approaches were able to maintain the percentage reduction.

\section{POLICIES}

The main idea of Policy-based Consumption Optimization is the definition of high level procedures policies - that will rule the behaviour of the power management mechanisms. The meaning of policy in this context is very simple: it is one or more rules that describe the action(s) to be taken when specific condition(s) exist. It can be expressed semantically as:

$$
\text { if (condition)then(action) }[\text { on(target })]
$$

The condition can include, but not limitted to, time constraints, calendar constraints, keyboard/mouse activity, processor activity, running processes footprint, performance registers content.

actions include actions related to changing the state of the computer in terms of energy consumption: turn-off, turn-on (Wake on LAN), suspend, hibernate.

Policies can be structured in global-level and userlevel. The former are of the responsibility of the manager and the latter of the responsibility of each user. The user must be able to specify that a computer will never be switched off, regardless of keyboard/mouse activity, and that the computer will never be switched off if an application program is running (Bray, 2006). In other words, users must have the freedom to choose switch off criteria that is best suited to their own work patterns (Newsham and Tiller, 1994).

\subsection{Power Probes}

The accuracy, or the overall quality, of a policy, depends on the use patterns of the organization's computers. A deep knowledge on how the computer is used, when it is being used, by which user, which processes are running, and others, provide valuable insight into how energy can be saved. To retrieve this information is necessary a probe (from now on, we will use the term agent to reference the probe) in each computer. The agent's responsibility is to read instrumentation parameters related to processes, performance registers, keyboard and mouse activity, and to send it to a central database. Aditionally, the agent is also responsible for applying policies, thus suspending, hibernating or shutting-down the computer. 


\section{Architecture}

Profiling computer energy use in an organization assumes a distributed power management mechanism, able of gathering the use pattern of several computers spread over the organization and of applying policies for energy saving. Each computer has a software agent, to perform instrumentation on the CPU's performance counters, keyboard and mouse activity, building the running processes and applications map and enforcing actions. Moreover, the agent can access mean and instant values for voltage, current, power and energy, provided by a hardware device, accessible through USB. The instrumentation data is sent to a centralized server, for persistence and data mining.

The hardware device is custom made and is installed in serial with the power supply. It has several internal registers, which can be read through USB. These values are used to correlate with the instrumentation values of the performance counters. This will allow to get the consumption scenario in several computers using a single power meter.

The communication between the agents and the central server is performed through Web Services, providing multi-platform and multi-language services for persistence, queries and management.The server also provides a Web based interface, to allow the definition, modification and removal of power saving policies.

\subsection{Power Probe - Agent}

From a power consumption point of view, computers are intrinsically heterogeneous. Differences in CPU technology, manufacturer, graphics, number of hard disk drives and so on make the process of profiling power consumption a difficult task. Moreover, without solid information about all the computers in the organization it is not possible to act, in terms of enforcing power saving policies.

Agents provide a common interface to obtain instrumentation values of the computer as well as providing a means to act on it. In other words, each agent periodically sends information related to the computer power consumption and enforces policies received from the central server.

Each agent has a set of sensors, modules responsible for getting and processing samples. For the purpose of the work presented in this paper, sensors monitor running processes, perform periodic samples on CPU performance counters, receive indication of keyboard and mouse activity, perform samples on the external power meter. Sensors also have a policy engine, responsible for running and enforcing policies.
The communication between agents and the policy server is performed through Web Services. This provides a interoperable communication mechanism, independent of the operating system, network technology and languages used.

\subsection{Policy Server}

The policy server is a central repository for power saving policies and power consumption history. It also provides a Web based user interface, to allow defining, updating and removing policies. It is developed in a multitier approach, isolating the persistence layer from the presentation layer with a business layer.

The business layer exports Web Services based interfaces, to allow remote operation on the server. Each module is encapsulated in an Enterprise Java Bean (EJB), preventing a direct access to the data in the database. In the work presented in this paper, the following modules are available: user and resources managament, policy definition, samples repository.

The user and resources management is related to the authentication and authorization of users in the organization. Each user is associated with an access list, which describe the resources he is allowed to control through the definition of policies. If a user tries to define a policy for a computer he has no authorization, the operation fails and the attempt is logged, for later inspection.

The policy definition module allows the user to write policies to the resources which he has access to. The policy, after validated, is stored in the database, through the persistence layer. Then the user has the possibility of activating it, which translates to sending the policy to the target agents. Deactivating the policy implies the removal of the agent in which it is running.

Finally, the samples module is responsible for receiving and storing the samples from the agents installed in the organization's computers. It also provides some statistics to the authorized users, to allow optimizing policies according to the power saving potential.

\subsection{User Behaviour}

The most important factor for saving energy is user behaviour, such as turning off devices at night or enabling power management (Newsham and Tiller, 1994; Webber et al., 2005). By providing adequate information as well as adequate tools, which can automate many of the user actions, it is possible to influence energy consumption reduction to a greate extent. 
Each agent has the possibility to popup a message, providing non-intrusive information about the power saving history in his computer. The most simple message is "You do not have power management activated in this computer. Do you want to turn it on? It would not have any impact on your work.". A more elaborate example: "Your computer will disconnect one hour after 8 p.m. However, the computer is usually idle after 5 p.m. Changing the policy would save 3 hours of energy consumption".

We believe that a discrete number of messages for month would appeal effectivelly to the user behaviour, thus contributing to reducing the overall expense.

\section{CONCLUSIONS}

Office equipment, in particular computers, demand a significant amount of power, contributing to the overall energy consumption. Profiling the power consumption of an organization require a distributed architecture, based on remote agents and a centralized server. The agents are responsible for instrumentation of computers, and the central server is responsible for storing and processing the information sent by the agents. Based on the energy profile, it is possible to define power saving policies, rules of behaviour for power management mechanisms, to be enforced by each agent.

The information from all the agents provide a valuable insight into how computers all over the organization use energy, as well as an opportunity to optimize power consumption based on the typical demand.

In this paper, we present and architecture for profiling and enforcing policies. It is based on a multitier application server with a Web frontend, and a web services middleware, to cope with the diversity and heterogeneity of enterprises' networks and systems.

\section{REFERENCES}

Ashok, R., Chheda, S., and Moritz, C. A. (2004). Coupling compiler-enabled and conventional memory accessing for energy efficiency. ACM Trans. Comput. Syst., 22:180-213.

Bray, M. (2006). Review of computer energy consumption and potential savings.

Diniz, B., Guedes, D., Meira, J. W., and Bianchini, R. (2007). Limiting the power consumption of main memory. In Proceedings of the 34th annual international symposium on Computer architecture, pages 290-301, San Diego, California, USA. ACM.
Flautner, K. and Mudge, T. (2002). Vertigo: automatic performance-setting for linux. SIGOPS Oper. Syst. Rev., 36:105-116.

Flinn, J. and Satyanarayanan, M. (1999). Powerscope: A tool for profiling the energy usage of mobile applications. In Proceedings of the Second IEEE Workshop on Mobile Computing Systems and Applications, page 210.

Hewlett-Packard, Microsoft, Intel, Technologies, P., and Toshiba (2006). Advanced configuration and power interface specification.

Hom, J. and Kremer, U. (2005). Inter-program optimizations for conserving disk energy. In Proceedings of the 2005 international symposium on Low power electronics and design, pages 335-338, San Diego, CA, USA. ACM.

Hsu, C. H. and Kremer, U. (2003). The design, implementation, and evaluation of a compiler algorithm for cpu energy reduction. ACM SIGPLAN Notices, 38:38-48.

Isci, C. and Martonosi, M. (2003). Runtime power monitoring in high-end processors: Methodology and empirical data. In Proceedings of the 36th annual IEEE/ACM International Symposium on Microarchitecture. IEEE Computer Society Washington, DC, USA.

Li, X., Li, Z., David, F., Zhou, P., Zhou, Y., Adve, S., and Kumar, S. (2004). Performance directed energy management for main memory and disks. In Proceedings of the 11th international conference on Architectural support for programming languages and operating systems, pages 271-283, Boston, MA, USA. ACM.

Newsham, G. and Tiller, D. (1994). The energy consumption of desktop computers: measurement and savings potential. Industry Applications, IEEE Transactions on, 30:1065-1072.

Qadeer, W., Rosing, T. S., Ankcorn, J., Krishnan, V., and Micheli, G. D. (2003). Heterogeneous wireless network management. In Proc. Wksp. Power Aware Computer Systems (PACS). Springer.

Su, H., Qiu, P., and Qiu, Q. (2004). Esacw: an adaptive algorithm for transmission power reduction in wireless networks. In Proceedings of the 2004 international symposium on Low power electronics and design, pages 82-85, Newport Beach, California, USA. ACM.

Webber, C. A., Roberson, J. A., McWhinney, M. C., Brown, R. E., Pinckard, M. J., and Busch, J. F. (2005). Afterhours power status of office equipment in the usa. Journal: Energy (the International Journal); Journal Volume: 31; Journal Issue: 14; Related Information: Journal Publication Date: 11/2006.

Weiser, M., Welch, B., Demers, A., and Shenker, S. (1996). Scheduling for reduced cpu energy. KLUWER INTERNATIONAL SERIES IN ENGINEERING AND COMPUTER SCIENCE, pages 449-472.

Zedlewski, J., Sobti, S., Garg, N., Zheng, F., Krishnamurthy, A., and Wang, R. (2003). Modeling hard-disk power consumption. FAST2003 - 2nd USENIX Conference on File and Storage Technologies. 